\title{
Dynamic Bandwidth and Wavelength Allocation Scheme for Next-Generation Wavelength-Agile EPON
}

\author{
Lin Wang, Xinbo Wang, Massimo Tornatore, Hwan Seok Chung, Han Hyub Lee, \\ Soomyung Park, and Biswanath Mukherjee
}

\begin{abstract}
Recently, the IEEE 802.3 Ethernet Working Group has classified three architectures for the nextgeneration Ethernet passive optical network (NG-EPON). They are called single-scheduling domain (SSD) PON, multi-scheduling domain (MSD) PON, and wavelength-agile (WA) PON, and they differ based on how a group of optical network units (ONUs) share a wavelength. Existing dynamic bandwidth and wavelength allocation (DBWA) schemes for conventional EPON can be applied to MSD-PON and SSDPON, but not WA-PON. This is because WA-PON is a new architecture with full flexibility where a flexible number of wavelengths can be assigned to one ONU, and multiple ONUs can transmit at the same time. In this work, we develop a mathematical model and a novel DBWA scheme for transmission scheduling in WA-PON. However, as WA-PON incurs penalties in terms of delay and power consumption when an ONU activates its transmissions on new wavelengths, a trade-off between energy saving and data-transfer latency reduction needs to be carefully addressed when performing transmission scheduling. So, we develop a power-consumption model and modify the proposed DBWA scheme to enhance the energy efficiency of WA-PON. Finally, we conduct simulation experiments for performance evaluation of the three PON architectures in terms of latency and packet loss ratio. We quantitatively investigate the influence of various parameters, such as the number of ONU transceivers and ONU buffer size, on WA-PON latency and packet loss ratio, and we evaluate the energy efficiency gain of the modified DBWA scheme.
\end{abstract}

Index Terms-Dynamic bandwidth allocation; Energy efficiency; NG-EPON; Performance evaluation; WA-PON.

\section{INTRODUCTION}

$\mathbf{T}$ he recent Cisco visual network index [1] reports that the amount of IP data handled by access networks is expected to realize sustained increases and will exceed 190 exabytes by 2018, on pace to reach 500 exabytes by 2020 . Such increase of bandwidth demands is stimulating new deployments of passive optical networks (PONs), due to

Manuscript received September 29, 2016; accepted January 3, 2017; published February 24, 2017 (Doc. ID 277562).

L. Wang (e-mail: amlwang@ucdavis.edu), X. Wang, M. Tornatore, and B. Mukherjee are with the University of California, Davis, California 95616, USA.

M. Tornatore is also with Politecnico di Milano, Italy.

H. S. Chung, H. H. Lee, and S. Park are with the Electronics and Telecommunications Research Institute, South Korea.

https://doi.org/10.1364/JOCN.9.000B33 their desirable features, such as high capacity, low latency, and cost efficiency.

Among current PON technologies, Ethernet PON (EPON) with $1 \mathrm{Gbit} / \mathrm{s}$ wavelength has been a marketleading optical access technology for several years. However, as 1G-EPON cannot satisfy the envisioned access data-rate growth due to its limited wavelength capacity, an IEEE 802.3 ad hoc [2] Working Group was created for nextgeneration EPON (NG-EPON). Recently, a new standard was released [2] stating that NG-EPON should operate at an aggregate data rate of $10 \mathrm{Gbit} / \mathrm{s}$ per wavelength with coverage of up to $100 \mathrm{~km}$.

A generic PON system consists of an optical line terminal (OLT) located at the central office and connected to multiple optical network units (ONUs) through a tree- or ring-based optical distribution network. Depending on the targeted logical connectivity, NG-EPON can support different architectures, including dedicated-fiber PON, timedivision multiplexing (TDM) PON, wavelength-division multiplexing (WDM) PON, and time and wavelengthdivision multiplexing (TWDM) hybrid PON. In particular, in hybrid PON, a set of wavelengths is shared over time by different ONUs instead of being dedicated as in WDMPON, and this improves the efficiency of network-resource usage.

Depending on how bandwidth is managed over various wavelengths, hybrid PON can be classified as singlescheduling domain (SSD) PON, multi-scheduling domain (MSD) PON, and wavelength-agile (WA) PON [2]. In this study, we investigate dynamic bandwidth and wavelength allocation (DBWA) schemes for these three hybrid PONs. DBWA is a mechanism implemented in OLTs to dynamically allocate bandwidth over various wavelengths in terms of time windows for data transmission at each ONU in upstream. Each ONU can transmit data only on its assigned wavelength(s) during the allocated time window to avoid conflict with other ONUs. In MSD-PON, each ONU can transmit on only one upstream wavelength at a time, and many ONUs can transmit simultaneously on different wavelengths, as shown in Fig. 1(a). In SSD-PON, each ONU must transmit on all upstream wavelengths, as shown in Fig. 1(b); hence, ONUs cannot transmit simultaneously. These two hybrid PON solutions were recognized to be sufficient under conventional scenarios. 


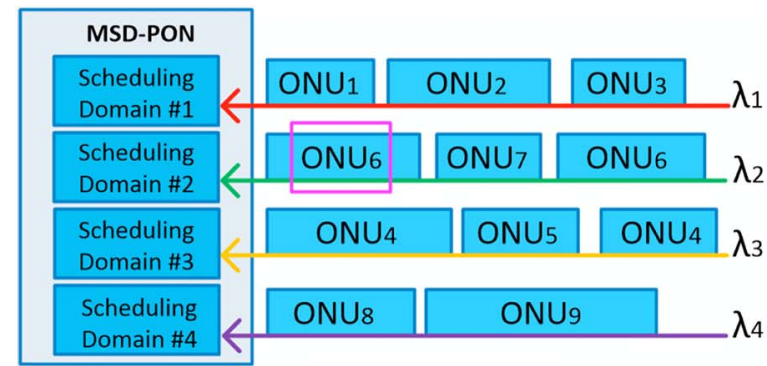

(a)

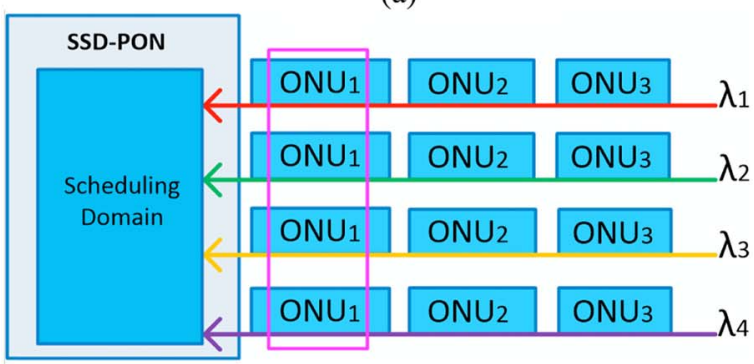

(b)

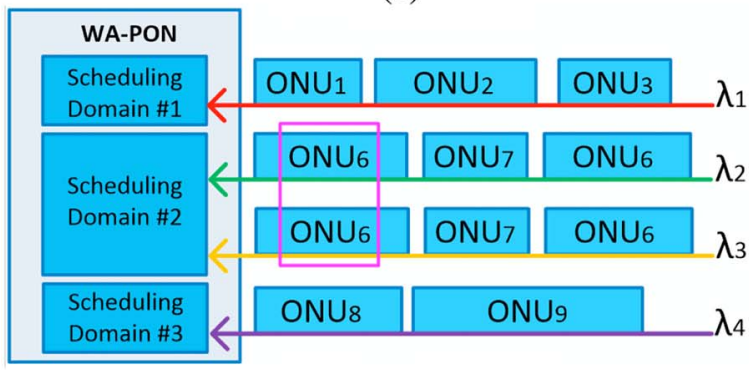

(c)

Fig. 1. Illustrations of three hybrid PON solutions: (a) MSD-PON, (b) SSD-PON, and (c) WA-PON.

However, new trends are being observed in users' traffic behavior in access networks, e.g., 1) business and residential users have different busy hours, which means only a few ONUs require large bandwidth at a certain time of the day, and the ONU with large bandwidth requirements might change during the day, and 2) traffic distribution is very skewed, with, e.g., real deployment scenarios where $10 \%$ of subscribers consume around $50 \%$ of all data traffic $[3,4]$. Therefore, NG-EPON should be designed for a more complex scenario [5-9], where "elephant flow" from, e.g., a cellular base station coexists with "mice flow" from, e.g., a machine-to-machine (M2M) communication.

To better accommodate such traffic diversity among ONUs, WA-PON has recently been proposed by the IEEE 802.3 Ethernet Working Group as another hybrid PON solution. WA-PON supports full flexibility of wavelength assignment and sharing, as any number of wavelengths can be assigned to any ONU at a certain time, and many ONUs can transmit simultaneously as long as their assigned wavelengths are not overlapped, as shown in Fig. 1(c). In this figure, note that $\mathrm{ONU}_{6}$ has much more data to transmit than $\mathrm{ONU}_{1}$ and $\mathrm{ONU}_{8}$, so we assign two wavelengths to $\mathrm{ONU}_{6}$ and one wavelength each to $\mathrm{ONU}_{1}$ and $\mathrm{ONU}_{8}$. Note that, in this example, $\mathrm{ONU}_{6}, \mathrm{ONU}_{1}$, and $\mathrm{ONU}_{8}$ can transmit at the same time, while $\mathrm{ONU}_{6}$ and $\mathrm{ONU}_{7}$ can share the same wavelengths without time overlapping.

Therefore, WA-PON can handle temporary traffic surges of certain ONUs by allocating large time windows on multiple wavelengths to reduce the transmission time, instead of forcing network operators to increase investment on an infrastructure with higher rates per wavelength or to scale their network according to the sum of peak data rates of all ONUs. However, existing dynamic bandwidth and wavelength allocation (DBWA) schemes cannot be directly applied to WA-PON, and, to the best of our knowledge, there is no work on quantitatively comparing the performance of the three hybrid PON solutions.

Moreover, today there is a growing interest in reducing the energy consumption and associated cost of the access network [10-17]. Energy modeling studies of the Internet have highlighted how, in the short-to-medium time period, energy consumption is overwhelmingly dominated by the access network, particularly by the ONU at subscriber premises [16]. So, WA-PON, as a candidate solution for NG-EPON, should also be energy efficient. When an ONU is lightly loaded, it can occupy fewer wavelengths to reduce power consumption. However, when an ONU is heavily loaded, more wavelengths should be assigned to it to reduce data-transfer latency. However, transmitting on multiple wavelengths needs to activate more lasers, which increases power consumption. Therefore, DBWA for WAPON should make a trade-off between energy saving and reduction of data transfer latency ("latency" for short), which is defined as the duration from the instant when a packet enters the buffer at an ONU to the instant when it arrives at the OLT. Note that minimizing energy consumption can be done in such a way that the system can still provide a reasonably low average delay.

In this study, we first introduce the details of three hybrid PON architectures. Then, we focus on WA-PON by 1 ) mathematically modeling the optimal transmission scheduling using an integer linear program (ILP) when traffic demands are known, 2) designing a novel DBWA scheme for online operation in WA-PON, and 3) developing a power consumption model for WA-PON and modifying the proposed DBWA to enhance the energy efficiency for WA-PON. Finally, we conduct simulation experiments to study 1) the performance gains of WA-PON enabled by our proposed DBWA scheme compared with the other two hybrid PONs in terms of latency and packet loss ratio; 2 ) the impact of various parameters, e.g., number of $\mathrm{ONU}$ transceivers and ONU buffer size, on the performance of WA-PON; and 3) energy-efficiency gain of the modified DBWA scheme.

The rest of the study is organized as follows. Section II introduces the three hybrid PONs: SSD-PON, MSD-PON, and WA-PON. In Section III, we mathematically model the transmission scheduling in WA-PON using an ILP, and design a novel DBWA scheme for WA-PON. In Section IV, we improve the proposed DBWA to devise an energy-efficient scheme. In Section V, we conduct simulation experiments for numerical study. Section VI concludes this work. 


\section{Three Hybrid PON Architectures}

In a PON, the OLT is the device with access to downstream wavelengths, and it transmits data without needing arbitration. In the upstream direction, it is again the OLT that grants access to wavelengths to the ONUs, allowing an ONU to transmit its buffered data. Hybrid PON can be implemented according to three different architectures, depending on the way the OLT arbitrates the access to the wavelengths.

\section{A. $M S D-P O N$}

In MSD-PON, an OLT grants an ONU access only to a single wavelength at a time to transmit the buffered data. As an ONU is allowed to transmit data on only one wavelength at a time, multiple ONUs can transmit simultaneously on different wavelengths, as shown in Fig. 1(a). The specific duration of such a grant to the ONU, i.e., the transmission time window, depends on the DBWA scheme employed by the OLT. As MSD-PON is similar to current TWDM-PON, efficient DBWA schemes already developed for TWDM-PON can be applied to MSD-PON, e.g., earliest finish time (EFT) [18]. With EFT, when an OLT receives a REPORT message (which specifies the required data size and ONU state information) from an ONU, the OLT will assign the wavelength with the earliest finish time to the specific ONU. The OLT then informs the specific ONU of the grant information through a Gate message including starting time, allocated wavelength, and size of granted bandwidth.

\section{B. SSD-PON}

In SSD-PON, the OLT grants an ONU access to all available upstream wavelengths at the same time. No other ONU is allowed to transmit during the same time window, as shown in Fig. 1(b). The duration of such a grant to the given ONU depends again on the specific DBWA scheme. SSD-PON is similar to TDM-PON, so any efficient dynamic-bandwidth-allocation scheme for TDM-PON can be applied in SSD-PON, e.g., interleaved polling with adaptive cycle time (IPACT) [19]. With IPACT, when the $k$ th ONU is transmitting Ethernet frames in the upstream, the OLT informs the $(k+1)$ th ONU of grant information, including starting time and size of granted bandwidth. The $(k+1)$ th ONU may start transmission before the $k$ th ONU, but the first bit of the $(k+1)$ th ONU cannot arrive at the OLT until the $k$ th ONU completes. Note that, with IPACT, an ONU with heavy traffic load may monopolize the upstream wavelength for a long time, so packets from other ONUs may be excessively delayed. To solve this problem, an upper bound $B_{\max }$ is set to limit the maximum bandwidth allocated to each $\mathrm{ONU}$ in a cycle. Our study considers a $10 \mathrm{Gbit} / \mathrm{s}$ data rate in NG-EPON, and we set $B_{\max }$ as infinite, i.e., the OLT grants to an ONU the exact amount of bytes reported in its last REPORT message. Because WA-PON can assign multiple wavelengths to
ONUs with high traffic load, transmitting all data at one time exploits the flexibility of WA-PON and improves the quality of service (QoS) of networks.

\section{WA-PON}

WA-PON has been recently proposed by the IEEE 802.3 Ethernet Working Group [2]. In WA-PON, an OLT grants access to a flexible number of wavelengths to a specific ONU to transmit its buffered data. To support multiplewavelength assignment of WA-PON, an ONU may need more than one transceiver [10]. In addition, a DBWA scheme is needed to arbitrate upstream transmissions to avoid collisions and effectively use flexible sharing in WA-PON. However, most existing DBWA schemes, which are designed for other PON architectures [20-24], are not suitable for WA-PON. In fact, existing DBWA schemes consider how to grant bandwidth on only a fixed number of assigned wavelengths for each ONU, while in WA-PON a flexible number of upstream wavelengths can be assigned to an ONU. Thus, WA-PON needs a new DBWA scheme that can address three main questions: 1) How many wavelengths should be assigned to an ONU based on the control frame (REPORT)? 2) Which wavelengths should be assigned to the specific ONU? and 3) How should the transmission windows be distributed over the assigned wavelengths? The authors of [20] proposed a DBWA scheme for WA-PON in which a preset threshold is introduced. If the uplink data in an ONU exceeds the preset threshold value, the ONU will send a message to the OLT to request another wavelength. However, this DBWA scheme still does not consider fully flexible wavelength assignment and sharing in WA-PON, because each ONU can use at most two wavelengths to transmit at the same time [20].

\section{Mathematical Model and DBWA Design}

In this section, we mathematically model the transmission scheduling scheme in WA-PON using an ILP, and design a novel DBWA scheme for WA-PON.

\section{A. Mathematical Model}

\section{1) Given}

- $W$ : set of wavelengths $\left\{\lambda_{1}, \lambda_{2}, \ldots, \lambda_{w}\right\}$.

- $W_{\max }$ : maximum usable wavelengths for one ONU (depending on available transceivers in an ONU).

- $R_{i}$ : arrival time of the $i$ th request (the time dimension is modeled through discrete time slots).

- $G_{i}$ : requested window size of the $i$ th request, measured in time slots needed to transmit buffered data of this request over one wavelength.

- $M$ : a very large positive value.

\section{2) Integer Variables}

- $S_{i, w}$ : transmission start time for the $i$ th request on $\lambda_{w}$. 
- $T_{i, w}$ : grant (number of time slots) for the $i$ th request on $\lambda_{w}$.

- $R e q F_{i}$ : transmission finish time of the $i$ th request.

\section{3) Binary Variables}

- $\delta_{i, j, w}$ : whether the transmission start time of the $i$ th request is earlier than the transmission start time of the $j$ th request on $\lambda_{w}$ (i.e., if $S_{i, w}<S_{j, w}$, then $\delta_{i, j, w}=1$; otherwise $\delta_{i, j, w}=0$ ).

- $K_{i, w}$ : whether the $i$ th request uses $\lambda_{w}$.

\section{4) Objective}

Minimize total delay for all requests:

Minimize $\sum_{i=1}^{N}\left(\operatorname{Req} F_{i}-R_{i}\right)$.

\section{5) Constraints}

Subject to the following constraints:

$$
\begin{gathered}
\sum_{1 \leq w \leq|W|} T_{i, w} \geq G_{i} \quad \forall i, \\
T_{i, w} \leq M * K_{i, w} \quad \forall i, w, \\
T_{i, w} \geq K_{i, w} \quad \forall i, w, \\
\sum_{1 \leq w \leq|W|} K_{i, w} \leq W_{\max } \quad \forall i, \\
S_{i, w} \geq K_{i, w} * R_{i} \quad \forall i, w, \\
S_{i, w} \leq M * K_{i, w} \quad \forall i, w, \\
\delta_{i, j, w}+\delta_{j, i, w}=1 \quad \forall i, j, w, \\
S_{i, w}-S_{j, w} \leq M * \delta_{j, i, w} \quad \forall i, j, w, \\
S_{j, w} \leq M *\left(1-\delta_{i, j, w}+2-K_{i, w}-K_{j, w}\right) \\
\forall i, j, w, \\
S_{i, w}+T_{i, w}-T_{i, w} \\
\forall i, j, w, \\
S_{j, w}+T_{j, w}-S_{i, w} \leq M *\left(1-\delta_{j, i, w}+2-K_{i, w}-K_{j, w}\right) \\
\forall i, w .
\end{gathered}
$$

Equation (1) ensures that the sum of transmission windows on all assigned wavelengths should be equal to the size of the $i$ th request. Equations (2) and (3) assign a value to $K_{i, w}$ : if the $i$ th request occupies $\lambda_{w}$, then $K_{i, w}=1$; otherwise $K_{i, w}=0$. Equation (4) ensures that the number of wavelengths assigned to the $i$ th request does not exceed the maximum number of wavelengths supported by an ONU. Equation (5) ensures that the transmission start time for the $i$ th request on $\lambda_{w}$ should not be earlier than its arrival time. Equation (6) ensures that, if the $i$ th request does not occupy $\lambda_{w}\left(K_{i, w}=0\right)$, then $S_{i, w}$ must be zero. Otherwise, if $K_{i, w}=1$, we assume $S_{i, w}$ and $S_{j, w}$ are bounded by a large constant $M$. For any two requests, $i$ and $j$, occupying the same wavelength $w$, Eqs. (7) and (8) ensure that either $\delta_{i, j, w}=1$, if the transmission start time of the $i$ th request is earlier than that of the $j$ th request on $\lambda_{w}$, or $\delta_{j, i, w}=1$ (i.e., $S_{i, w}>S_{j, w}$ ).
For any two requests, $S_{i, w}$ and $S_{j, w}$, occupying $\lambda_{w}$, Eqs. (9) and (10) ensure that any two transmission windows on the same wavelength are not overlapping. When one (or both) requests $R_{i}$ and $R_{j}$ do not occupy $\lambda_{w}\left(K_{i, w}=0\right.$ or $\left.K_{j, w}=0\right)$, then we do not have to consider whether they are overlapping. In this case, Constraints (9) and (10) are deactivated (i.e., they hold always, irrespective of $S_{i, w}$ and $S_{j, w}$ ), since the right-hand side of the constraints take a value $M$ large enough to be always higher than the left-hand side.

Now, assume that both requests $R_{i}$ and $R_{j}$ occupy $\lambda_{w}$ ( $K_{i, w}=1$ and $K_{j, w}=1$ ). Then, one of Eqs. (9) or (10) is activated according to the values of $\delta_{i, j, w}$ and $\delta_{j, i, w}$. In particular, Eq. (9) is activated when $\delta_{i, j, w}=1$ (i.e., when $S_{i, w}<S_{j, w}$ ), in which case Eq. (9) becomes

$$
S_{i, w}+T_{i, w} \leq S_{j, w}
$$

ensuring that the transmission window used by two requests on the same wavelength do not overlap. When $\delta_{i, j, w}=1$, then $\delta_{j, i, w}=0$, and Eq. (10) is deactivated, since Eq. (10) becomes

$$
S_{j, w}+T_{j, w}-S S_{i, w} \leq M,
$$

which holds always irrespective of $S_{i, w}$ and $S_{j . w}$. In a similar manner, Eq. (10) is activated when $\delta_{j, i, w}=1$, (i.e., when $S_{i, w}>S_{j . w}$ ) and Eq. (9) is deactivated. Thus, Eqs. (9) and (10) ensure that the transmission windows assigned to different requests on the same wavelength do not overlap.

Equation (11) assigns to $R e q F_{i}$ the value of the latest transmission finish time for the $i$ th request. In this way, minimizing the sum of all $R e q F_{i}-R_{i}$ is equivalent to transmitting all requests as soon as possible so as to minimize the total delay. Also, it can compress all transmission windows on all wavelengths as tight as possible so as to utilize wavelength resources efficiently.

The number of variables and constraints used by the ILP formulation depends on the number of wavelengths, maximum usable wavelengths per ONU, and requests. In the worst case (where each request occupies all wavelengths), the formulation needs $2 W(N-1) N$ Boolean $\delta_{i, j, w}$ variables, $W(N-1) N$ equalities for Eq. (7), and $2 W(N-1) N$ inequalities for Eqs. (8)-(10). The rest of the formulation uses: $W N$ Boolean and integer variables for $K_{i, w}, S_{i, w}$, and $T_{i, w} ; N$ constraints for Eqs. (1) and (4); and $W N$ for Constraints (2), (3), (5), and (6). So, the complexity of variables is $O\left(W N^{2}\right)$, where $W$ is the number of wavelengths, and $N$ is the number of requests. The complexity of constraints is also $O\left(W N^{2}\right)$.

\section{B. Novel DBWA Scheme for WA-PON}

When the traffic profile is not known a priori, or is subject to statistical uncertainty, the OLT needs to make online decisions for transmission scheduling in WA-PON, instead of executing a pre-planned schedule (as in the static case described above). The design of an online DBWA scheme bears the objectives of (1) minimizing the average delay, or (2) reducing the packet loss ratio. The inputs to the scheduling strategy are the data size requested by an ONU and the current state, i.e., occupations of the wavelengths. 
To address the two objectives stated above, we propose a novel DBWA scheme for WA-PON, called water-filling DBWA (WF-DBWA). The idea is to consider the wavelength resource as a depression storage, where the storage bed of each stage is the finish time of the last reservation on each wavelength, and the water to be filled is the time window required by an ONU to transmit the buffered data at the data rate of one wavelength. The objective is to minimize the water surface, while filling all the water (required time window) into the depression storage, just like gravity leads to the lowest water surface. In this way, the transmission of each request will finish as soon as possible, thus reducing the delay.

In Algorithm 1, we present the pseudocode of WFDBWA. We suppose that the OLT receives a control message (REPORT) that specifies data size $\left(G_{i}\right)$ needed by the $i$ th request from the $k$ th ONU. In Line 1 , we record and sort all wavelengths $W=\left\{\lambda_{0}, \lambda_{1}, \ldots, \lambda_{w}\right\}$ in ascending order of $S_{i, w}$, which is the presumed transmission start time for the $i$ th request on $\lambda_{w}$. $S_{i, w}$ is calculated by the formula in Line 1 , where $f_{w}$ is the finish time of the last transmission on $\lambda_{w}, t$ is current time, $R d_{k}$ is the round-trip propagation delay of the $k$ th ONU, and $t_{c}$ is the processing delay of the control frame. Then, we initialize the allocated time window $C_{i}$ as zero, the reserved wavelengths set $A_{i}$ as empty, and the pointer to a wavelength on which the time window will be allocated, $w$, pointing at the wavelength with the lowest $S_{i, w}$ (Line 2).

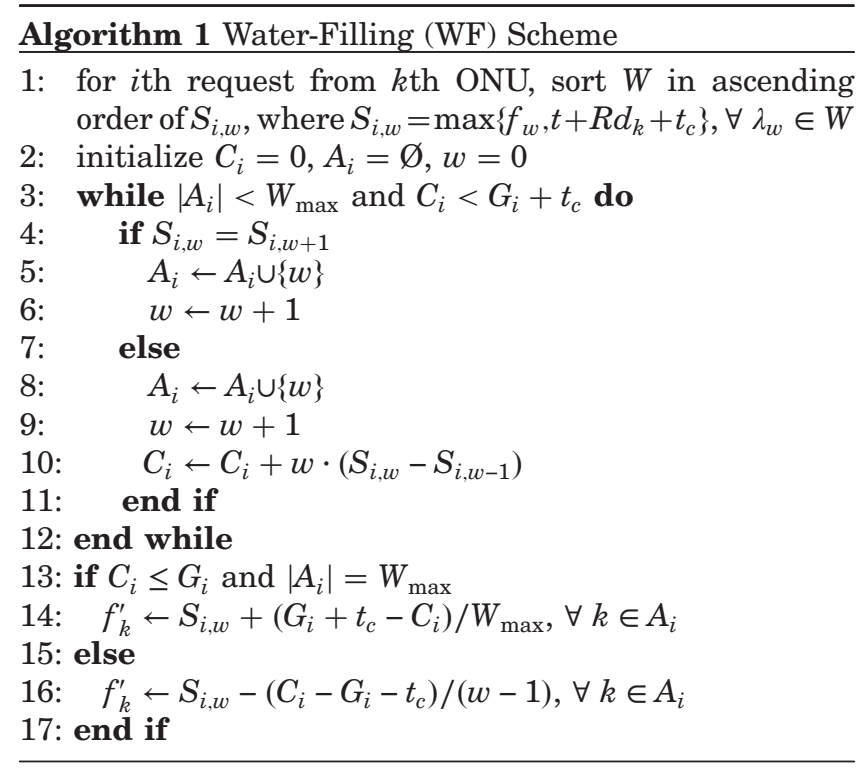

We fix the maximum number of wavelengths $W_{\max }$ that can be assigned to an ONU, because an ONU has a limited number of transceivers. We iteratively reserve new wavelengths and allocate more time windows on reserved wavelengths for the $k$ th ONU until $C_{i}$ is no less than the required time window $G_{i}$, or the number of reserved wavelengths reaches the limit $W_{\max }$ (Lines 3-12). At each iteration, if $S_{i, w}$ of $\lambda_{w}$ is equal to $S_{i, w+1}$ of the next $\lambda_{w+1}$, we just add $\lambda_{w}$ into $A_{i}$ (Lines 4-6) and renew the pointer $w$. If $S_{i, w}$ is lower than $S_{i, w+1}$, we not only add $\lambda_{w}$ into $A_{i}$ and increase $w$ by 1 , but also renew $C_{i}$ by allocating time windows on all reserved wavelengths (Lines 7-11), similar to pouring more water to raise the water surface. The while loop terminates when the number of reserved wavelengths reaches the limit or the allocated time window is larger than or equal to the required time window. In the former case, we may not allocate enough time window for the $i$ th request from the $k$ th $\mathrm{ONU}$, so we need to equally allocate the remaining time window on all reserved wavelengths in $A_{i}$ (Line 14). In the latter case, we may allocate redundant transmission windows for the $i$ th request, so we need to equally redo the unnecessary time window by renewing the finish time of each reserved wavelength (Lines 15-17).

The time complexity of Algorithm 1 is dominated by the sorting in Line 1 , which can be $O(W \log W)$, where $W$ is the total number of wavelengths. Operations in Lines 3-12 and Lines $13-17$ both have at most $W_{\max }$ iterations. If wavelengths are maintained in a minimum heap according to its $S_{i, w}$ value, the time complexity of Algorithm 1 can be $O\left(W_{\max } \log W\right)$.

In Fig. 2, an example of WF-DBWA is shown with $W_{\max }=4$. In Step 1, as the first and second wavelengths have the same start time, the OLT will assign time windows on $\lambda_{1}$ and $\lambda_{2}$ until their new finish time equals the start time of $\lambda_{3}$. In Step 2, the OLT continues to assign time windows on $\lambda_{1}, \lambda_{2}$, and $\lambda_{3}$ until they reach the start time of $\lambda_{4}$. Since $W_{\max }=4$, which means the OLT can assign no more than four wavelengths to the ONU, and the total required time window $G_{i}$ has not been satisfied, we need to allocate more time windows on the reserved four wavelengths, as shown in Step 3.

\section{ENERGY-EFFICIENT DBWA SCHEME FOR WA-PON}

\section{A. Novel Energy Efficient DBWA Scheme}

Although WA-PON could minimize the data transfer latency for an ONU by allocating the required time window on multiple wavelengths, this approach has some shortcomings. To transmit on multiple wavelengths, every ONU in WA-PON needs to be equipped with a large number of transceivers, each responsible for transmitting and receiving data on one or a (pair of) wavelength(s).

In this study, we assume a vertical-cavity surface-emitting laser (VCSEL) [10] as such a transceiver employed by an ONU. A VCSEL is able to stay in sleep mode to save energy when it is not transmitting data. However, when an OLT assigns wavelengths to an ONU, VCSELs needs to be switched from sleep mode to active mode, and this operation (activation) incurs a power-consumption impulse lasting for a short time, during which no data can be transmitted, but it consumes extra power [10]. Therefore, we modify WF-DBWA to consider the cost of activating VCSELs, and we call this new approach energy-efficient WF-DBWA (EEWF-DBWA).

EEWF-DBWA can decide whether it is desirable to activate more wavelengths to transmit data and how many additional wavelengths to activate. For example, when overall traffic load is light and the available wavelengths' 

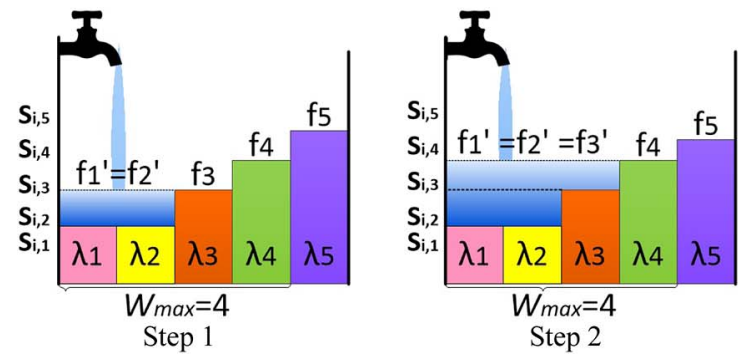

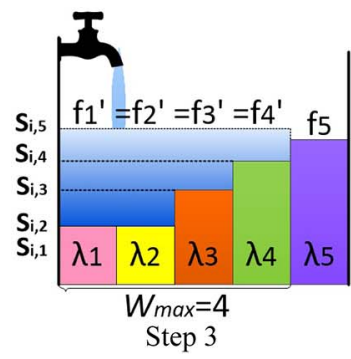

Fig. 2. WF-DBWA example.

bandwidth is sufficient in networks, an ONU can transmit its buffered data in a timely manner even using a small subset of the available wavelengths. In this situation, we do not need to assign wavelengths in a greedy manner as in WF-DBWA; instead, we will assign "just-enough" wavelengths to save energy as long as we do not increase overall latency significantly.

On the other hand, when the traffic load of an ONU becomes comparable to its interface capacity, i.e., total data rates of wavelengths, the delay and packet loss ratio can rapidly increase, and we refer to this situation as "splash" (to use a water-filling metaphor) in this study. When the network begins to splash, if only a few wavelengths (e.g., one) are assigned to an ONU, its latency will increase significantly, because it will take a long time to transmit buffered packets and many packets will be queued (even dropped) at the ONU due to the large traffic demand. Therefore, activating more VCSELs at heavy load is more valuable than at light load, and we need to effectively address the trade-off between saving energy and reducing data transfer latency.

In EEWF-DBWA, we introduce a threshold variable $V_{\text {thresh, }}$ which is adaptive to the offered load of the network (i.e., the ratio between the traffic demand generated per second by all ONUs and the total capacity of all wavelengths):

$$
V_{\text {thresh }}=\left\{\begin{array}{cc}
1-L / p, & 0<L<p \\
0, & \text { otherwise }
\end{array},\right.
$$

where $L$ is current offered traffic load and $p$ denotes the offered load at which the splash begins. The $p$ value is adjustable with the evolution of traffic, i.e., we can set it as the load where the splash begins to reflect current traffic characteristics and control the network during its lifetime. The use of $V_{\text {thresh }}$ is shown in Lines 8 and 9 in Algorithm 2, which represents the main difference between EEWFDBWA and WF-DBWA schemes. In Line 8, when deciding to assign a new wavelength, we consider two aspects: (1) adding one more wavelength must significantly help to reduce the transmission finish time, namely, the ratio of the already-assigned time window to the entire required time window must be smaller than $V_{\text {thresh }}$ (i.e., the rest of the unassigned data should be large enough), and (2) we value energy saving more only when the offered load is light. When $L$ is smaller than $p$, with growing offered load, $V_{\text {thresh }}$ is decreasing, which increases the possibility of assigning more wavelengths as explained in (1). If the offered load is larger than $p, V_{\text {thresh }}$ is set as zero to stop the energy-saving mechanism. We can set up different values for $V_{\text {thresh }}$ during a day by adjusting the $p$ value.

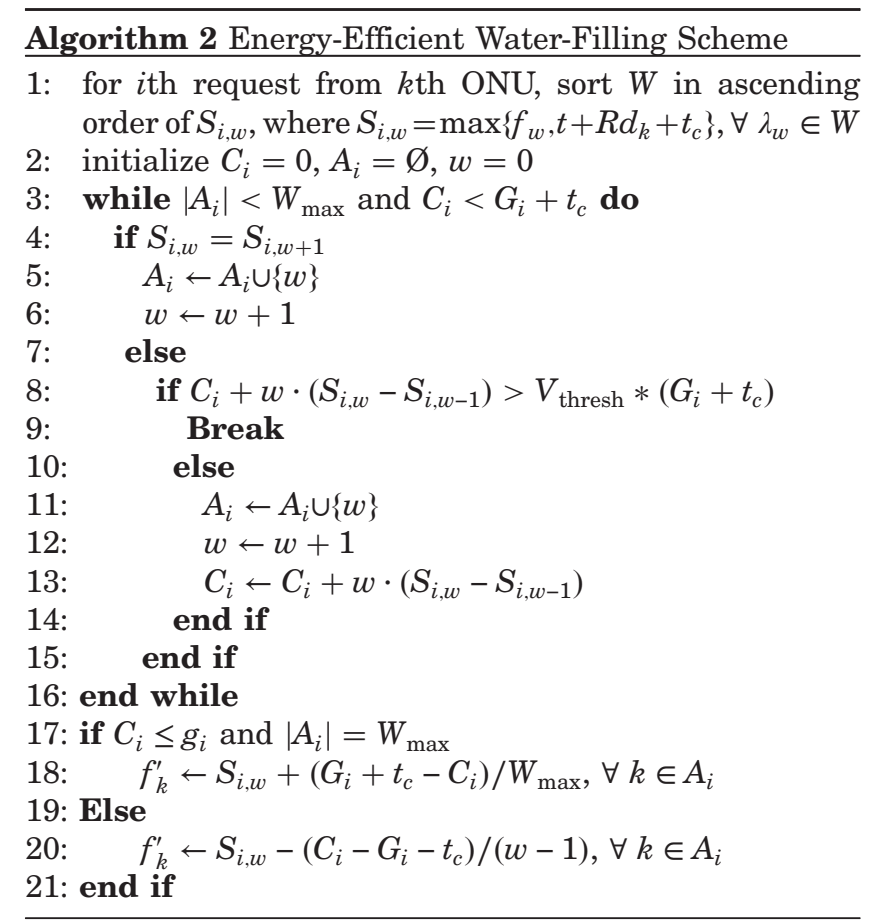

\section{B. Power-Consumption Model}

In our model, we assume the power consumption of a VCSEL-ONU changes only between active mode [i.e., both transceiver (TX) and receiver (RX) are powered up] and sleep mode (i.e., both TX and RX are powered down). Power consumption values considered in our work are listed in Table I [10]. $P_{\text {active }}$ and $P_{\text {sleep }}$ are power consumptions when an ONU operates in active mode and sleep mode, respectively. $T_{\text {sett }}$ and $T_{\text {rec }}$ are setting time and recovery time of the VCSEL-ONU, respectively. The energy cost $E_{i}$ for the $i$ th transmission request is calculated as

$$
E_{i}=\left|A_{i}\right| * P_{\text {active }} *\left(T_{\text {sett }}+T_{\text {rec }}\right)+P_{\text {active }} * G_{i},
$$

where $\left|A_{i}\right|$ is the number of reserved wavelengths, which equals the number of VCSEL laser transmitters we need to turn on. $P_{\text {active }}$ multiplied by $T_{\text {sett }}$ plus $T_{\text {rec }}$ returns the energy in joules required to switch a laser from sleep 
TABLE I

Power Consumption and Transition Times of 10 Gbits/s VCSEL-ONU

\begin{tabular}{lccccc}
\hline Mode & TX Block $(\mathrm{W})$ & RX Block $(\mathrm{W})$ & $P_{\mathrm{ONU}}(\mathrm{W})$ & $T_{\text {sett }}(\mu \mathrm{s})$ & $T_{\text {rec }}(\mu \mathrm{s})$ \\
\hline Active & 0.134 & 3.85 & 3.984 & 0 & 0 \\
Sleep & 0 & 0.75 & 0.75 & 0.33 & 2 \\
\hline
\end{tabular}

mode to active mode. $P_{\text {active }}$ multiplied by $G_{i}$ is the energy in joules required to transmit all buffered data of an ONU.

\section{Illustrative Numerical Examples}

Our performance evaluation of the DBWA schemes for WA-PON considers three cases. First, we compare the dynamic WF-DBWA scheme with the static ILP model, which provides the optimal scheduling. Then, we simulate three hybrid PON architectures to assess advantages of WA-PON in terms of packet loss ratio and average delay, and analyze the influence of different parameters on WA-PON. Finally, we evaluate the benefits of EEWFDBWA compared with WF-DBWA.

\section{A. Static Case}

To reflect real Internet traffic, we generate self-similar traffic by aggregating multiple sub-streams, each consisting of alternating Pareto-distributed ON/OFF periods, with a Hurst parameter of 0.8 . In the following simulations, we have used the traffic generator as in [18]. The size of Ethernet frames is uniformly drawn from 64 to 1518 bytes.

We conduct simulation experiments to first evaluate the performance of WA-PON in the static case, where the arrival time and size of bursts are given $a$ priori. The network consists of eight ONUs and four wavelengths, and each ONU has two transmitters (i.e., two maximum usable wavelengths). The capacity of a wavelength is $10 \mathrm{Gbit} / \mathrm{s}$, which gives a total capacity of $40 \mathrm{Gbit} / \mathrm{s}$. The offered load is the ratio between the expected number of frames generated per second by the eight ONUs and the total capacity of the four wavelengths (i.e., $40 \mathrm{Gbit} / \mathrm{s}$ ). For a given offered load, we use the self-similar traffic generator described above to generate 50 bursts as input data of the ILP, and we iterate 100 independent instances to guarantee that all the plotted values have a $95 \%$ confidence interval not larger than $5 \%$. We use CPLEX software to solve the ILP and Java to realize the WF-DBWA scheme.

In Fig. 3, we compare the average delay obtained by the ILP model and the WF-DBWA for WA-PON at different offered loads. We see that, at low loads (i.e., up to load equal to 0.7 , when splash occurs), WF-DBWA performs almost the same as ILP, WF-DBWA has $4.7 \%$ more delay at an offered load of $0.1,13 \%$ more delay at an offered load of 0.7 , and 9.6\% more delay than static ILP on average between offered loads of 0.1 and 0.7 . As the offered load grows, the difference of delay between WF-DBWA and ILP increases because, at higher load, it is more challenging for the OLT to dynamically allocate the wavelength resource efficiently

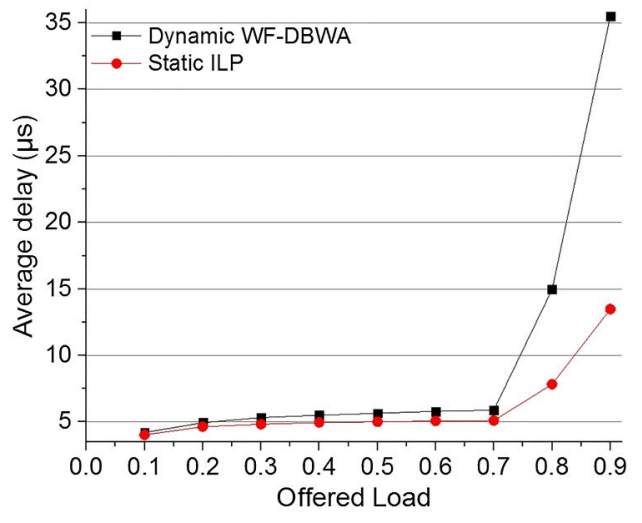

Fig. 3. Average delay of the static ILP model and the WF-DBWA model.

to each request. As in real networks, the offered load is generously smaller than 0.7 , so WF-DBWA should perform efficiently in practice.

\section{B. Dynamic Case}

We compare the three scheduling strategies for NGEPON, i.e., MSD-PON, SSD-PON, and WA-PON, in a dynamic case, where future traffic is unknown. We use a generic tree topology with 128 ONUs transmitting on eight wavelengths. Considering that business and residential users have different traffic load, we classify 128 ONUs into two groups. One group contains business ONU users occupying $10 \%$ of the total ONUs (i.e., 12) and generates relatively high traffic demand, while the other group consists of residential users who generate relatively low traffic. We assume the traffic demand generated by business users is 10 times higher than that of residential users. The buffer size of each ONU is 10 Mbytes. Distances between the OLT and the ONUs are distributed between 500 and $20 \mathrm{~km}[2,3]$.

In NG-EPON, each wavelength has a bit rate of $10 \mathrm{Gbit} / \mathrm{s}$, which gives a total capacity of $80 \mathrm{Gbit} / \mathrm{s}$. The normalized offered load for the whole network varies during the simulation from 0.05 to 1 . The offered load is the ratio between the expected packet bursts generated per second by all ONUs (i.e., including business and residential users) connected to an OLT and the total capacity of eight wavelengths (i.e., $80 \mathrm{Gbit} / \mathrm{s}$ ). At offered load 0.1, the traffic load for each ONU is $64 \mathrm{Mbit} / \mathrm{s}$, where the average traffic demand for business users is $347 \mathrm{Mbit} / \mathrm{s}$ and for residential users is 34.7 Mbit/s. The time required for the transmission of control frames, $t_{c}$, is $5 \mathrm{~ns}$. We apply the EFT scheme in MSDPON [18] and the modified IPACT scheme in SSD-PON [19], while WA-PON uses the proposed WF-DBWA scheme. The performance metrics of interest are the average delay (i.e., time from generation of a packet at the ONU until its reception at the OLT) and the overall packet loss ratio.

Figure 4 shows that the average delays of MSD-PON, SSD-PON, and WA-PON increase as the offered load grows, as more packets are queued in the buffers of ONUs. The average delay of MSD-PON starts to splash at load 0.6, 


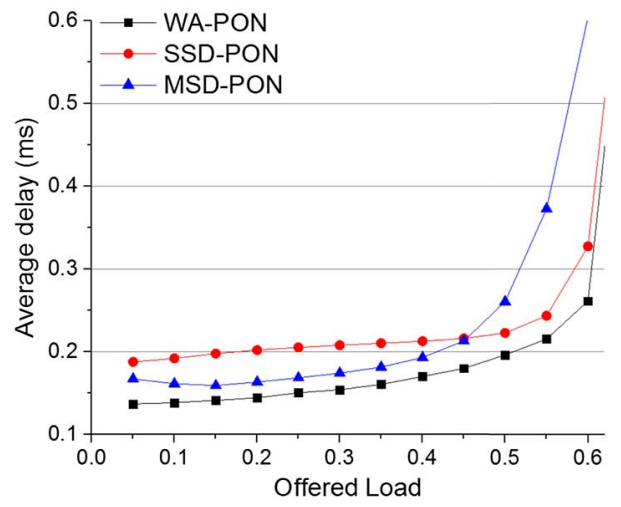

Fig. 4. Average delay of SSD-PON, MSD-PON, and WA-PON.

but WA-PON can prevent from splashing until load 0.65 and has lower delay compared to MSD-PON at any offered load. This is because the OLT in WA-PON can allocate multiple wavelengths (i.e., large bandwidth) to reduce the average delay, and the WF-DBWA scheme can efficiently decide how many and which wavelengths should be assigned to them, while MSD-PON can assign each ONU with only one wavelength. WA-PON also achieves a lower delay compared with SSD-PON at any offered load because, in SSD-PON, no more than one ONU is allowed to transmit at the same time, which means all other ONUs must wait until the current ONU finishes its transmission.

Figure 5 shows the packet loss ratio of the three architectures. We can see that WA-PON can reduce the packet loss ratio compared with SSD-PON before splash, and confirm that WA-PON can postpone the splash compared with MSD-PON, which suggests that WA-PON can tolerate more traffic and performs the best among the three scheduling strategies.

In Figs. 6 and 7, we study the impact of the $W_{\max }$ value on the delay and packet loss ratio for WA-PON, where $W_{\max }$ is the maximum number of wavelengths that can be assigned to an ONU. Note that SSD-PON can be considered a special case of WA-PON in which $W_{\max }$ equals the number of all wavelengths (i.e., eight in our case), and MSD can be considered a special case with $W_{\max }=1$. By increasing the $W_{\max }$ value from 2 to 7 , we can notice that our

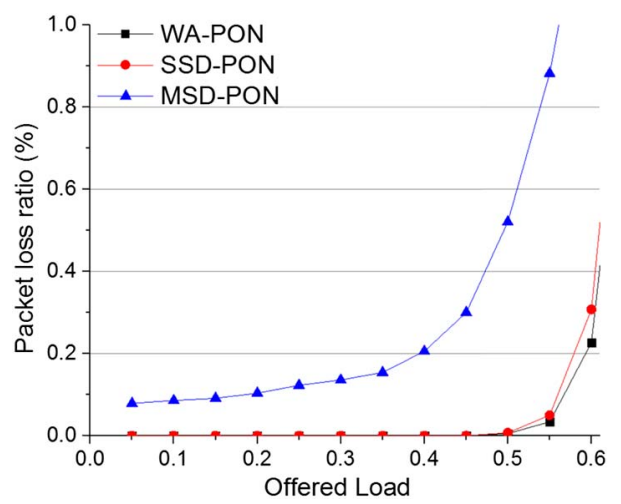

Fig. 5. Packet loss ratio of SSD-PON, MSD-PON, and WA-PON.

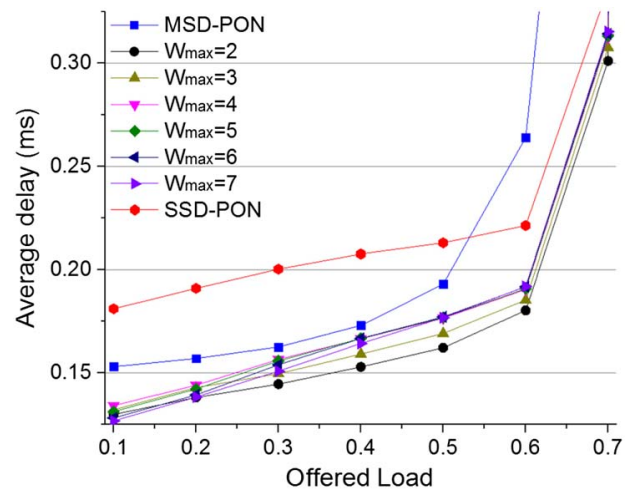

Fig. 6. Average delay of WA-PON with different $W_{\max }$ values.

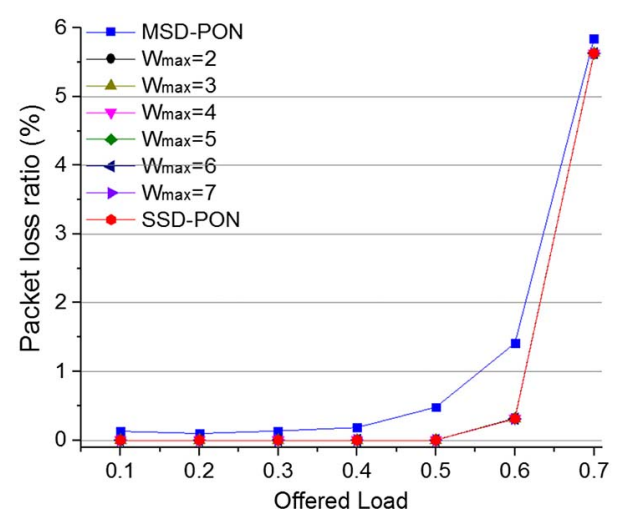

Fig. 7. Packet loss ratio of WA-PON with different $W_{\max }$ values.

WF-DBWA performs best when $W_{\max }=2$. This is because, if we set $W_{\max }$ to be very large, then an ONU will occupy too many wavelengths and starve other ONUs, which increases packet delay in other ONUs and leads to larger opportunity for dropping packets in other ONUs. However, the optimal $W_{\max }$ changes with different values of buffer size and total number of wavelengths. For example, when there are eight wavelengths with $50 \mathrm{MB}$ buffer size for each ONU, the optimal $W_{\max }$ is 4 , because with increasing buffer size there can be much larger required data so that one ONU needs to transmit on more wavelengths to reduce the transmission delay. When there are six wavelengths with $50 \mathrm{MB}$ buffer size for each ONU, the optimal $W_{\max }$ is 3 , because when the total number of wavelengths decreases, to avoid starving other ONUs with light load, optimal $W_{\max }$ should be smaller.

In Figs. 8 and 9, we study the impact of buffer size for an ONU on delay and packet loss ratio in WA-PON. On the one hand, increasing buffer size can help decrease the packet loss ratio, as shown in Fig. 9, but the improvement is not noticeable. However, increasing buffer size will lead to more average delay (see Fig. 8), especially for high loads, because a larger buffer allows a longer queue of data waiting to be transmitted and thus longer delay for a packet. So, network operators need to make a trade-off between the delay and packet loss ratio when they deploy a larger buffer in WA-PON. 


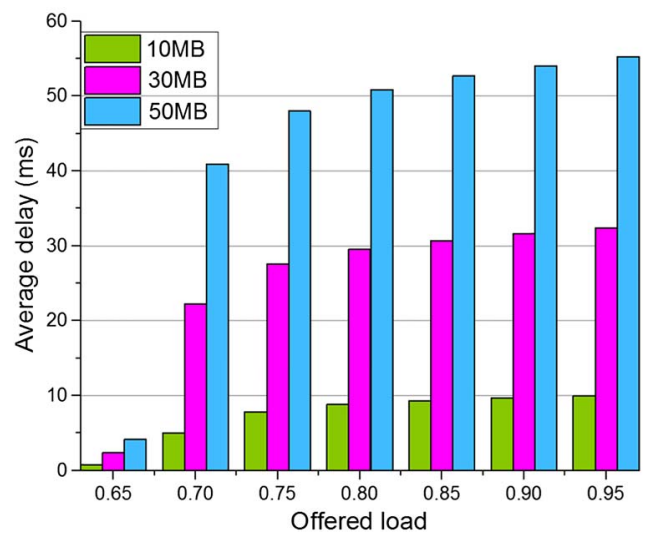

Fig. 8. Average delay of MSD-PON with different buffer sizes.

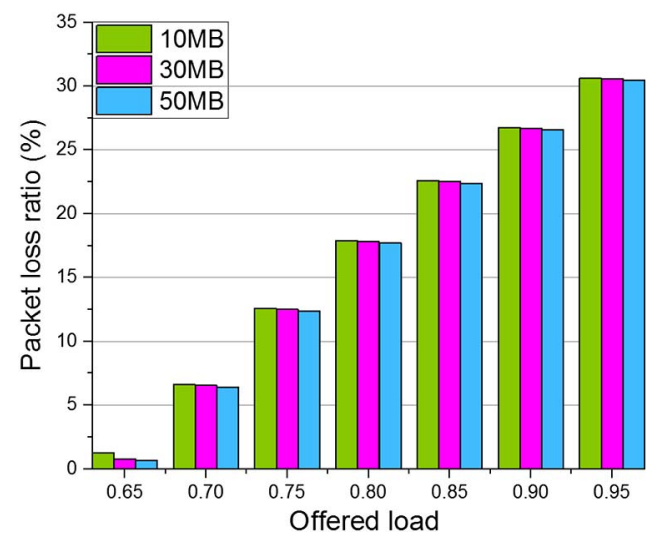

Fig. 9. Packet loss ratio of MSD-PON with different buffer sizes.

\section{Energy-Efficiency Study}

In Fig. 10, we compare EEWF-DBWA with WF-DBWA with respect to energy efficiency, which is the ratio between throughput and energy consumption in joules. We set $W_{\max }$ as 2 so that EEWF-DBWA will not increase the average delay much compared with WF-DBWA. The energy efficiency of EEWF-DBWA increases with growing offered load. Before the splash point (0.55), EEWF-DBWA is

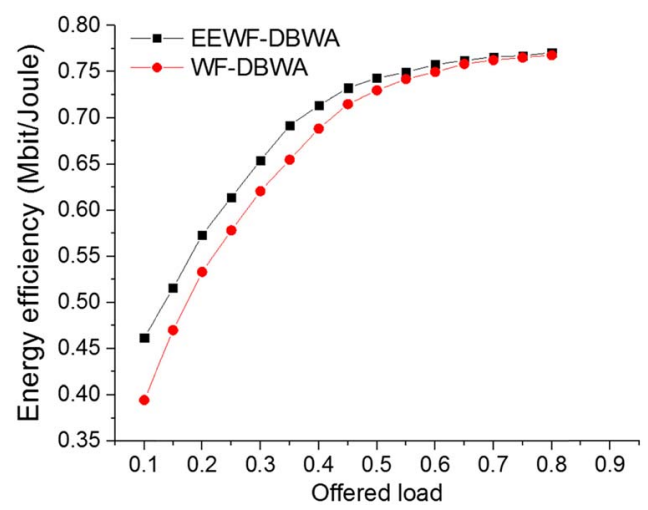

Fig. 10. Energy efficiency of WF-DBWA and EEWF-DBWA.

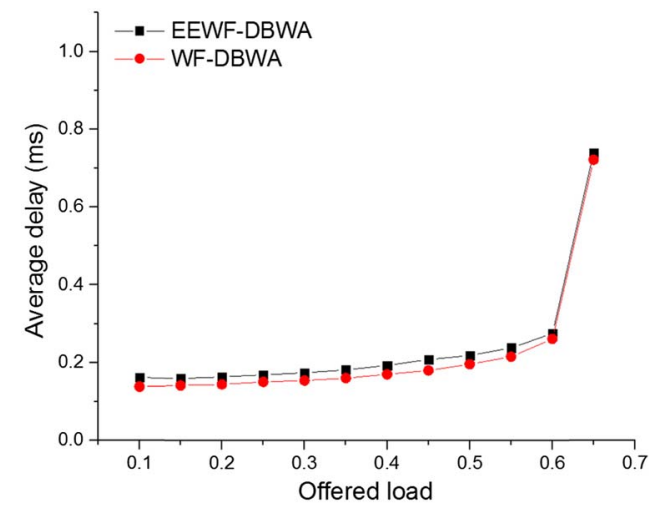

Fig. 11. Average delay of EEWF-DBWA and WF-DBWA.

$17 \%$ more energy efficient for offered load 0.1 , and $6 \%$ more energy efficient than WF-DBWA on average between offered loads of 0.1 and 0.6, which means EEWF-DBWA can send more bits per joule. With increasing value of $W_{\text {max }}$, EEWF-DBWA will give higher energy efficiency than WF-DBWA while having longer average delay. Therefore, network operators need to make a trade-off between energy efficiency and average delay by setting an appropriate $W_{\max }$. When offered load is light, EEWF-DBWA uses fewer wavelengths, which may slightly increase data transfer latency, but save considerable energy, thus leading to better energy efficiency than WF-DBWA. However, with increasing offered load, $V_{\text {thresh }}$ decreases according to Eq. (12). Therefore, the energy efficiency plays a less important role so that EEWF-DBWA performs more similar to WF-DBWA.

Figure 11 compares EEWF-DBWA and WF-DBWA with respect to average delay. When the offered load is smaller than the $p$ value, the delay of EEWF-DBWA is reasonably a little higher than that of WF-DBWA, because EEWFDBWA limits the possibility of assigning more wavelengths to one ONU to save power consumption. However, when the offered load is larger than the $p$ value, EEWF-DBWA performs very close to WF-DBWA. This is because the network begins to splash, and to guarantee the network QoS, energy efficiency is sacrificed.

\section{Conclusion}

We studied a hybrid PON architecture called wavelength-agile PON (WA-PON). We developed an integer linear program (ILP) for optimal transmission scheduling in WA-PON when traffic demands are given. A novel dynamic bandwidth allocation (DBWA) scheme was also designed for online operation in WA-PON, called water-filling DBWA (WF-DBWA). We conducted simulation experiments to benchmark WF-DBWA compared with ILP, and results show that WF-DBWA can achieve near-optimal performance with ILP when traffic load is low. We also compared the performance of WA-PON with two other hybrid PON architectures, i.e., MSD-PON and SSD-PON. Simulation results show that WA-PON can perform better than the other two architectures in terms of average delay and 
packet loss ratio. In addition, WA-PON can tolerate heavier traffic load to support more bandwidth-consuming subscribers (e.g., base stations). Additionally, we proposed an energy-efficient WF-DBWA scheme, which performs better at saving energy than WF-DBWA.

\section{ACKNOWLEDGMENT}

This work was supported by an Institute for Information \& Communications Technology Promotion (IITP) grant funded by the South Korean government (MSIP) (B013215-1004, SDN based wired and wireless converged optical access networking).

\section{REFERENCES}

[1] Cisco, "Visual networking index: Forecast and methodology, 2015-2020," June 2016.

[2] IEEE 802.3 Ethernet Working Group, "IEEE 802.3 industry connections feasibility assessment for next generation of EPON," Mar. 2015.

[3] L. Shi, S. Lee, H. Song, and B. Mukherjee, "Energy-efficient long-reach passive optical network: A network planning approach based on user behaviors," IEEE Syst. J., vol. 4, no. 4, pp. 449-457, 2010.

[4] E. Dahlman, G. Mildh, S. Parkvall, J. Peisa, J. Sachs, and Y. Selen, "5G radio access," Ericsson Rev., vol. 6, pp. 2-7, 2014.

[5] X. Wang, S. Thota, M. Tornatore, H. S. Chung, H. H. Lee, S. Park, and B. Mukherjee, "Energy-efficient virtual base station formation in optical-access-enabled cloud-RAN," IEEE J. Sel. Areas Commun., vol. 34, no. 5, pp. 1130-1139, 2016.

[6] X. Wang, S. Thota, M. Tornatore, S. S. Lee, H. H. Lee, S. Park, and B. Mukherjee, "Handover reduction via mobilityprediction-based VPON formation in optical-access-enabled cloud-RAN," in Optical Fiber Communication Conf. (OFC), Mar. 2015, paper Tu2E.2.

[7] X. Wang, S. Thota, M. Tornatore, S. S. Lee, H. H. Lee, S. Park, and B. Mukherjee, "Green virtual base station in opticalaccess-enabled cloud-RAN," in Proc. Int. Conf. Communications (ICC), June 2015, pp. 5002-5006.

[8] B. Kantarci and H. T. Mouftah, "Bandwidth distribution solutions for performance enhancement in long-reach passive optical networks," IEEE Commun. Surv. Tutorials, vol. 14, no. 3, pp. 714-733, 2012.

[9] J. Zhang, N. Ansari, Y. Luo, F. Effenberger, and F. Ye, "Nextgeneration PONs: A performance investigation of candidate architectures for next-generation access stage 1," IEEE Commun. Mag., vol. 47, no. 8, pp. 49-57, 2009.

[10] E. Wong, M. Mueller, M. P. Dias, C. A. Chan, and M. C. Amann, "Energy-efficiency of optical network units with vertical-cavity surface-emitting lasers," Opt. Express, vol. 20, no. 14, pp. 14960-14970, 2012.

[11] M. P. I. Dias, D. P. Van, L. Valcarenghi, and E. Wong, "Energyefficient framework for time and wavelength division multiplexed passive optical networks," J. Opt. Commun. Netw., vol. 7, no. 6, pp. 496-504, 2015.

[12] S. Lambert, J. Montalvo, J. A. Torrijos, B. Lannoo, D. Colle, and M. Pickavet, "Energy efficiency analysis of nextgeneration passive optical network (NG-PON) technologies in a major city network," in 15th Int. Conf. on Transparent Optical Networks (ICTON), June 2013, pp. 1-6.

[13] H. Mukai, "Energy efficient 10G-EPON system," in Optical Fiber Communication Conf. (OFC), Mar. 2013, paper OW3G.1.

[14] J. I. Kani, "Power saving techniques and mechanisms for optical access networks systems," J. Lightwave Technol., vol. 31, no. 4, pp. 563-570, 2013.

[15] J. I. Kani, S. Shimazu, N. Yoshimoto, and H. Hadama, "Energy-efficient optical access networks: Issues and technologies," IEEE Commun. Mag., vol. 51, no. 2, pp. S22-S26, 2013.

[16] J. Baliga, R. Ayre, K. Hinton, W. V. Sorin, and R. S. Tucker, "Energy consumption in optical IP networks," J. Lightwave Technol., vol. 27, no. 13, pp. 2391-2403, 2009.

[17] C. M. Machuca, E. Wong, M. Furdek, and L. Wosinska, "Energy consumption and reliability performance of survivable hybrid passive optical converged networks," in Photonics Networks and Devices, July 2016, paper NeTu3C.2.

[18] K. Kanonakis and I. Tomkos, "Improving the efficiency of online upstream scheduling and wavelength assignment in hybrid WDM/TDMA EPON networks," IEEE J. Sel. Areas Commun., vol. 28, no. 6, pp. 838-848, 2010.

[19] G. Kramer, B. Mukherjee, and G. Pesavento, "IPACT a dynamic protocol for an Ethernet PON (EPON)," IEEE Commun. Mag., vol. 40, no. 2, pp. 74-80, 2002.

[20] X. Cheng, Y. K. Yeo, C. Li, and X. Xu, "Wavelength-agile optical access networking system," Proc. SPIE, vol. 8310, 83100O, 2011.

[21] M. Mahloo, J. Chen, L. Wosinska, A. Dixit, B. Lannoo, D. Colle, and C. M. Machuca, "Toward reliable hybrid WDM/TDM passive optical networks," IEEE Commun. Mag., vol. 52, no. 2, pp. S14-S23, 2014.

[22] J. Zheng and H. T. Mouftah, "A survey of dynamic bandwidth allocation algorithms for Ethernet passive optical networks," Opt. Switching Netw., vol. 6, no. 3, pp. 151-162, 2009.

[23] B. Skubic, J. Chen, J. Ahmed, L. Wosinska, and B. Mukherjee, "A comparison of dynamic bandwidth allocation for EPON, GPON, and next-generation TDM PON," IEEE Commun. Mag., vol. 47, no. 3, pp. S40-S48, 2009.

[24] N. Radzi, S. K. Sadon, and M. H. Al-Mansoori, "Dynamic bandwidth allocation EPON survey," in IEEE Student Conf. Research and Development (SCOReD), 2013, pp. 149-153. 